\title{
Liquid metals surface temperature fields measurements with a two-colour pyrometer
}

\author{
Romain Monier $^{\mathrm{a}, \mathrm{b}, *}$, François Thumerel ${ }^{\mathrm{a}}$, Julien Chapuis ${ }^{\mathrm{a}}$, Fabien Soulié ${ }^{\mathrm{b}}$, Cyril Bordreuil ${ }^{\mathrm{b}}$ \\ ${ }^{\text {a } A R E V A ~ N P, ~ C e n t r e ~ T e c h n i q u e, ~ F r a n c e ~}$ \\ ${ }^{\mathrm{b}}$ Laboratoire de Mécanique et Génie Civil, Université de Montpellier, France
}

\begin{abstract}
The paper presents an apparatus to measure surface temperature distribution of liquid metals during fusion processes. The apparatus is based on dual wavelength radiation thermometry and is designed to measure temperature from 1500 to $3000 \mathrm{~K}$. The pyrometer is based on standard optical parts and indus-trial CCD cameras. Uncertainties are analysed on the base of the radiometric equations. To insure relative precision in the measurement, a calibration procedure is conducted on tungsten ribbon with known emissivity in the temperature range. This procedure is also used to discuss the influence of wavelength couples. To conclude, experimental measurements on pure laser melted materials are conducted to assess the validity of the apparatus and methodology.
\end{abstract}

Keywords:

Liquid metals, Two-colour pyrometer, Temperature fields, Solidification front, Laser melting, Thermography

\section{Introduction}

The monitoring of liquid phase behaviour and temperature is of major concern for joining technologies. Some joining processes use an energy beam (arc, laser,...) to melt one or several materials. The liquid metal exchanges heat with the solid part but also with the environment along interfaces that can evolve with time. The liquid phase behaviour is driven by different phenomena (Marangoni, buoyancy, Lorentz force,...) and the velocities inside the liquid phase can reach $2 \mathrm{~m} / \mathrm{s}$ in welding process, for example. Temperature monitoring allows to follow the stability of heat and mass transfer inside the liquid metals all along the process. Thermocouples have been used to record the thermal cycle for experimental purposes but it often requires an inverse method to reach temperature in the liquid. It can be poorly used for monitoring process. For processes which involve liquid metals non contact method are required. Radiative methods are used to estimate temperature fields of metals over their melting point. To be robust, a noncontact measurement method for fusion process must not be sensitive to emission of the beam energy source, fluid flow velocity, emissivity dependence on wavelength and temperature and free surface orientation and motion.

\footnotetext{
* Corresponding author at: Laboratoire de Mcanique et Gnie Civil, Universit de Montpellier, France.

E-mail address: romain.monier@areva.com (R. Monier).
}

For contact-less temperature measurement, the most widespread method is infra-red camera. The application is defined by the camera sensor type and the infrared spectral range used. If the material radiative properties are well known, its accuracy can be very high as [1] present in their review. But, this method main disadvantage is the need of emissivity value on the complete wavelength range used for data acquisition, on all the temperature range and sometimes with relative orientation of the sensor to the scanned surface. The emissivity of melted materials has been extensively studied [2] around the melting point. Wilthan et al. [3] measured the emissivity of iron over its melting point, but at fixed wavelength with a direct laser reflection method in a static configuration.

As emissivity is a constant problem in radiometric temperature measurements, Schöpp et al. [4] and Muller et al. [5] combined near infra-red high speed camera with a spectrometer. Spectrometer is used to perform a point-wise spectrum. Based on radiation theory and the measured spectrum, the temperature and emissivity are computed with the help of a least-square fitting. This punctual measurement is then used to calibrate the high speed camera and to reconstruct the surface temperature distribution. Schöpp et al. [4] used this method during welding process and his results demonstrate that the emissivity is independent of temperature in the weld pool. These methods seem to be very effective because they estimate temperature and emissivity during the process. The method seems difficult to apply as a monitoring process and 


\begin{tabular}{|c|c|c|c|}
\hline \multicolumn{4}{|c|}{ Nomenclature } \\
\hline$L_{\lambda}$ & monochromatic spectral radiance $\left(\mathrm{W} \mathrm{sr}^{-1} \mathrm{~m}^{-3}\right)$ & $\varepsilon_{\lambda}$ & emissivity at wavelength $\lambda$ \\
\hline$L_{\lambda}^{0}$ & monochromatic blackbody spectral radiance $\left(\mathrm{W} \mathrm{sr}-1 \mathrm{~m}^{-3}\right)$ & $\rho_{0}$ & electrical resistivity of tungsten at $\mathrm{T}=300 \mathrm{~K}(\Omega \mathrm{m})$ \\
\hline$h$ & Planck constant $(\mathrm{J} s)$ & $\beta$ & volumetric thermal expansion coefficient \\
\hline$c$ & light celerity $\left(\mathrm{m} \mathrm{s}^{-1}\right)$ & $R_{0}$ & electrical resistance of the tungsten ribbon at $\mathrm{T}=300 \mathrm{~K}$ \\
\hline$k_{B}$ & Boltzmann constant $\left(\mathrm{J} \mathrm{K}^{-1}\right)$ & & $(\Omega)$ \\
\hline $\mathrm{Ng}$ & gray scale level & $R(T)$ & electrical resistance of the tungsten ribbon at $\mathrm{T}(\Omega)$ \\
\hline a & $\begin{array}{l}\text { first constant of sensitivity compensation approxima- } \\
\text { tion }\end{array}$ & $\begin{array}{l}\left(f_{x}, f_{y}\right) \\
\left(c_{x}, c_{y}\right)\end{array}$ & $\begin{array}{l}\text { intrinsic camera parameter: focal length } \\
\text { intrinsic camera parameter: optical centre }\end{array}$ \\
\hline $\mathrm{b}$ & $\begin{array}{l}\text { second constant of sensitivity compensation approxi- } \\
\text { mation }\end{array}$ & $\begin{array}{l}p_{1}, p_{2} \\
k_{1}, k_{2}, k_{3}\end{array}$ & $\begin{array}{l}\text { tangential distortion parameters } \\
\text { radial distortion parameters }\end{array}$ \\
\hline c & $\begin{array}{l}\text { third constant of sensitivity compensation approxima- } \\
\text { tion }\end{array}$ & $\begin{array}{l}r \\
t_{\text {expo }}\end{array}$ & $\begin{array}{l}\text { distance between a point and the optical centre } \\
\text { exposure time (s) }\end{array}$ \\
\hline $\begin{array}{l}K_{\lambda_{1}, \lambda_{2}} \\
T \\
\lambda \\
C_{f}\end{array}$ & $\begin{array}{l}\text { sensitivity compensation factor } \\
\text { temperature }(K) \\
\text { wavelength }(\mathrm{nm}) \\
\text { correction factor }\end{array}$ & $S_{\lambda}$ & sensor sensitivity at wavelength $\lambda\left(\mathrm{W}^{-1} \mathrm{sr} \mathrm{m}^{3} \mathrm{~s}\right)$ \\
\hline
\end{tabular}

the setup always rises the problem of dependence of the emissivity relative to the orientation.

Another approach is to get reed of emissivity by measuring surface radiation on multiple wavelength and assuming an emissivity behaviour. Field measurements can be done with IR or NIR sensors. The main problem is to synchronise spatially and with time, two wavelength observations. Thevenet et al. [6], Yamazaki et al. [7] and Tanaka et al. [8] use a two-colour pyrometer with which surface radiation are acquired at two different wavelengths. The ratio of these two data reveals a ratio of the emissivity at these two wavelengths. The most common hypothesis is to consider them equal, it is the grey body hypothesis where the emissivity is assumed to be constant all along the considered spectrum. In some case, a non-grey compensation can be introduced in the analysis [9]. Some others, [10-12], use different emissivity behaviour law in function of the material. The key point for this method is to choose the right wavelengths and the associated emissivity behaviour. The influence of relative velocity is not often discussed for such method.

In the present work, a two-colour pyrometer is developed to measure surface temperature during fusion process. First, the theoretical radiation theory and the designed device are presented. Due to the device arrangement, geometrical and spectral calibration have to be conducted. The ratio of grey level is then related to a calibrated tungsten ribbon with known temperature and emissivity. Uncertainties are discussed based on the device arrangement and theoretical aspects. To assess the validity of our setup, some experiments are conducted on pure materials melted with a laser beam.

\section{Measurement method}

The two-colour pyrometry is a radiative measurement method based on the Planck's law:

$L_{\lambda}^{0}(T)=\frac{2 h c^{2}}{\lambda^{5}} \frac{1}{e^{\frac{h c}{2 k_{B} T}}-1}$

Based on the blackbody model, the spectral radiance $L_{\lambda}^{0}(T)$ is due to the emission of radiation by the surface of an opaque body at temperature $T$. The emission spectrum of an actual object differs from the blackbody spectrum. The difference between them is taken into account by the introduction of emissivity:

$L_{\lambda}(T)=\varepsilon_{\lambda}(T) L_{\lambda}^{0}(T)$
The emissivity $\varepsilon_{\lambda}(T)$ depends on wavelength and temperatures. Emissivity can also be influenced by various parameters such as: surface roughness that can increase emissivity of aluminium alloys for example [13], surface oxidation [14] or phase change (liquid/ solid) [2]. The direction of observation can also be an influential factor due to the non-isotropic emission of radiation [15].

The two-colour pyrometry method uses the Wien's approximation of Planck's law (1):

$L_{\lambda}(T)=\varepsilon_{\lambda}(T) \frac{2 h c^{2}}{\lambda^{5}} \frac{1}{e^{\frac{h c}{k_{B} T}}}$

This approximation (3) is valid for wavelength and temperature couples satisfying $\lambda T \leqslant 2898 \mu \mathrm{m} \mathrm{K}$. The target temperature $T$ of a material point can be then derived from the ratio of the radiance at two different wavelengths $\lambda_{1}$ and $\lambda_{2}$ :

$T=\frac{h c}{k_{B}} \frac{\left(\lambda_{1}-\lambda_{2}\right)}{\lambda_{1} \lambda_{2}} \frac{1}{\ln \left(\frac{\varepsilon_{\alpha_{2}} L_{\lambda_{1}} \lambda_{1}^{5}}{\varepsilon_{\lambda_{1}} L_{\lambda_{2}} \lambda_{2}^{5}}\right)}$

The calculation of the target temperature $T$ with Eq. (4) requires the determination of the ratio of the values of emissivity $\varepsilon_{\lambda_{1}}$ and $\varepsilon_{\lambda_{2}}$, which are difficult to identify in the case of liquid metals. Assuming that the emissivity does not differ within the selected wavelengths $\lambda_{1}$ and $\lambda_{2}$ (grey body behaviour), the target temperature can be obtained with (5):

$T=\frac{h c}{k_{B}} \frac{\left(\lambda_{1}-\lambda_{2}\right)}{\lambda_{1} \lambda_{2}} \frac{1}{\ln \left(C_{f} \frac{L_{\lambda_{1}} \lambda_{1}^{5}}{L_{k_{2}} \lambda_{2}^{5}}\right)}$

The correction factor $C_{f}$ is considered equal to one in most of the studied cases. Its value can be modified if the material radiative properties are well known.

As Eq. (5) shows, the two work wavelengths are major parameters in the two-colour pyrometer method. They have to be chosen in the infrared or near-infrared domains, over $780 \mathrm{~nm}$, but small enough to validate Wien's approximation. As the maximum targeted temperature is around $3000 \mathrm{~K}$ (liquid metals), wavelengths have to be under $1000 \mathrm{~nm}$. The selected wavelengths $\left(\lambda_{1}, \lambda_{2}\right)$ also directly influence the temperature measurement uncertainty (see Section 4). Indeed, the measurement uncertainty is directly proportional to a coefficient uncertainty: $\left|\frac{T \lambda_{1} \lambda_{2}}{C\left(\lambda_{2}-\lambda_{1}\right)}\right|$ with $C=\frac{h c}{k_{B}}$. Increasing the separation of the wavelength $\Delta \lambda=\lambda_{2}-\lambda_{1}$ reduces then the temperature measurement uncertainty. But the assumption of the 
grey body behaviour can be less valid for large value of separation $\Delta \lambda$. The choice of the couple of wavelengths $\left(\lambda_{1}, \lambda_{2}\right)$ is therefore a compromise between the choice of targeted and equipment materials, the grey body assumption and the measurement uncertainty. The choice of satisfying values of $\left(\lambda_{1}, \lambda_{2}\right)$ in order to insure a reasonable effect on uncertainty measurement and a sufficiently small separation $\Delta \lambda$ is presented in Section 4.

The two-colour pyrometry can be carried out for temperature field measurement by using two-dimensional sensors. A solution could consist in acquiring pictures of a same scene with two different cameras equipped with two different interference filters. This technical solution introduces two additional potential sources of uncertainties: a significant spatial correction of images in order to superpose them and a possible modification of emissivity because of the difference in the angles of optical axis of cameras, which can weaken the grey body assumption. To avoid such difficulties, the scene of interest has to be acquired with a same optical point of view. [16] used a specific optical device based on a divergence mirror, which splits the initial optical path in two optical paths. Monochromatic interference filters $\lambda_{1}$ and $\lambda_{2}$ are respectively used on each optical path. The monochromatic optical paths are then gathered on a same camera sensor (a different part of the sensor is dedicated to each monochromatic information).

In the present study, the two-colour pyrometer is composed of two identical near-infrared cameras equipped with two different monochromatic interference filters (Fig. 1). The scene of interest is captured by the two cameras with a common lens thanks to the use of a beam-splitter that allows an initial identical point of view and identical lengths of monochromatic optical paths. The synchronization of data acquisition is ensured by a common trigger for the two cameras. This experimental device, based on two cameras, can introduce geometrical and optical defects but also differences in the spectral response of sensors. The calibration procedures developed to minimize these possible defects are presented in Section 3 and the uncertainty analysis associated to the temperature measurement is discussed in Section 4.

\section{Correction and reference procedures}

The temperature measurement with the two-colour pyrometry is based on the ratio of two monochromatic pictures. The experimental device can introduce two main kinds of defects that can lower the temperature measurement accuracy. The first one is directly due to the optical system that can create distortions due

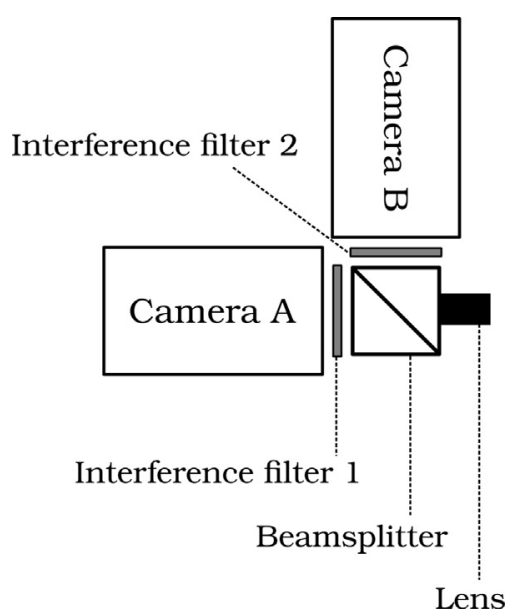

Fig. 1. Scheme of a two-colour pyrometer device. Cameras A and B are identical and a common trigger is used for data acquisition (cameras AVT GE 680). to the lens and different transformations due to the beamsplitter. A geometrical calibration procedure is proposed to correct these possible distortions. The second one concerns the differences in spectral response between the two cameras, which will be corrected with a spectral calibration procedure.

\subsection{Geometrical correction procedure}

Lens intrinsically introduces some radial distortions while misalignment between the optical axis and the camera sensor can give some tangential distortions. These distortions need to be corrected first to perform a further efficient image analysis. Dedicated open source code library (OpenCV) is used for these corrections [17].

Radial distortions can be approximated by a Taylor series expansion around the optical centre $(r=0)$ for which the radial distortion is null. Because of the symmetry, the correction function can be written with even power terms:

$$
\begin{aligned}
& x_{\text {corrected }}=x\left(1+k_{1} r^{2}+k_{2} r^{4}+k_{3} r^{6}\right) \\
& y_{\text {corrected }}=y\left(1+k_{1} r^{2}+k_{2} r^{4}+k_{3} r^{6}\right)
\end{aligned}
$$

Tangential distortions can be characterized by two other parameters, and the associated correction function can be expressed with equation:

$$
\begin{aligned}
& x_{\text {corrected }}=x+2 p_{1} y+p_{2}\left(r^{2}+2 x^{2}\right) \\
& y_{\text {corrected }}=y+2 p_{2} x+p_{1}\left(r^{2}+2 y^{2}\right)
\end{aligned}
$$

A calibrated 7 by 8 chessboard pattern is used to identify the distortions. Ten different orientations of the chessboard pattern were used to evaluate the whole set of coefficients: distortion correction coefficients $\left(p_{1}, p_{2}, k_{1}, k_{2}, k_{3}\right)$ and intrinsic coefficients $\left(f_{x}, f_{y}, c_{x}, c_{y}\right)$ of the optical device. This correction procedure shows that distortions are not significant; no perceptible difference appears with image subtraction analysis between raw frame and corresponding undistorted frame.

The associated corrections are then not taken into account in the whole process of temperature determination in order to increase its efficiency.

The beam-splitter and the opto-mechanical components can introduce slight changes in the two optical paths associated to camera A and camera B (Fig. 1). These changes in optical paths can create discrepancies between the two monochromatic frames $A$ and $B$ due to translation, rotation and scale changes. Frame A is considered as the reference picture. An affine transformation is applied to frame B to correct translation, rotation and scale differences. A given point with an initial position $(x, y)$ is located in position $\left(x^{\prime}, y^{\prime}\right)$ after correction:

$$
\left(x^{\prime}, y^{\prime}\right)=\left(M_{11} x+M_{12} y+M_{13}, M_{21} x+M_{22} y+M_{23}\right)
$$

with the affine matrix:

$$
M=\left(\begin{array}{lll}
M_{11} & M_{12} & M_{13} \\
M_{21} & M_{22} & M_{23}
\end{array}\right)
$$

The matrix elements $M_{11}, M_{12}, M_{21}, M_{22}$ correspond to rotation and scaling coefficients whereas $M_{13}, M_{23}$ are related to translation coefficients. No scale difference is observed between the undistorted frames A and B. The correction associated to this defect can be neglected and the affine matrix can be rewritten:

$$
M_{\text {mod }}=\left(\begin{array}{lll}
\cos \theta & -\sin \theta & M_{13} \\
\sin \theta & \cos \theta & M_{23}
\end{array}\right)
$$

The frame B is transformed according to:

$$
B^{\prime}=M_{m o d} B
$$


The elements $M_{13}, M_{23}$ and the rotation angle $\theta$ are computed by minimizing the correlation function $f(9)$ :

$f=1$

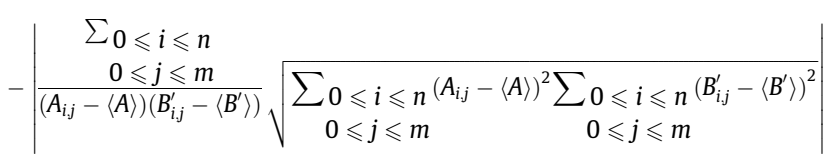

where $A$ and $B^{\prime}$ are the representative matrix of frames $A$ and $B$, and $n$ and $m$ are respectively the numbers of lines and rows of these matrix. The initialization of the minimization procedure is done by the choice of three points on each frame. Corrections are then applied on frame B in order to match with frame A.

\subsection{Spectral reference and conversion procedure}

The emission of radiation is converted by camera sensors into grey scale levels. Therefore, a conversion relation is required to associate grey scale levels $N g_{\lambda}$ with monochromatic radiance $L_{\lambda}$. As the grey scale levels are directly related to the photon energy received by the CCD camera sensor, the conversion relation can be written:

$N g_{\lambda}=L_{\lambda} t_{\text {expo }} S_{\lambda}$

where $t_{\text {expo }}$ is the exposure time and $S_{\lambda}$ the sensor sensitivity at wavelength $\lambda$. The exposure time is the same for the two cameras, the ratio of radiance can then be written:

$\frac{L_{\lambda_{1}}}{L_{\lambda_{2}}}=\frac{N g_{\lambda_{1}}}{N g_{\lambda_{2}}} \frac{S_{\lambda_{2}}}{S_{\lambda_{1}}}=\frac{N g_{\lambda_{1}}}{N g_{\lambda_{2}}} K_{\lambda_{1}, \lambda_{2}}$

with $K_{\lambda_{1}, \lambda_{2}}=\frac{S_{\lambda_{2}}}{S_{\lambda_{1}}}$ a sensitivity coefficient.

The sensitivity coefficient is determined thanks to a calibration procedure using a calibration source with identified radiance spectrum. The calibration source has to cover the temperature range of applications involving melting of metal and be made with material for which physical properties are well-known. For this purpose, tungsten is chosen because it is the metal having the highest melting temperature $(3695 \mathrm{~K})$ and its thermophysical and radiative properties are well-known $[18,19]$. The calibration procedure is based on the method proposed by [20] and uses a calibration lamp (lamp OSRAM WI 17G) with a controlled power supply (Fig. 2). By varying current and voltage in the appropriate range and considering Ohm's law and electrical resistivity behaviour, we can access to

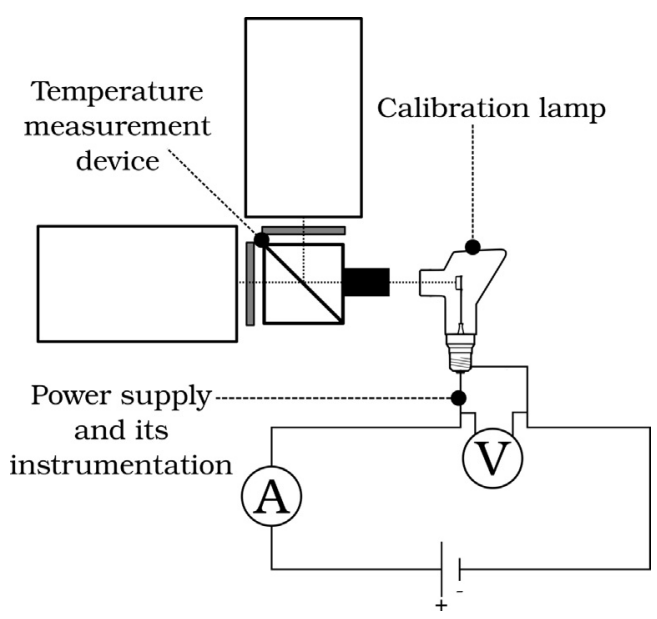

Fig. 2. Calibration set-up and instrumentation. the evolution of electrical resistance of tungsten filament in relation with temperature:

$\frac{R(T)}{R_{0}}=\frac{\rho(T)}{(1+\beta) \rho_{0}}$

The relation (12) can be fitted with a second order polynomial form, from which it is possible to calculate the temperature $T$ :

$\frac{R(T)}{R_{0}}=2.8420718 \times 10^{-7} T^{2}+0.00466128 T-0.52427113$

The monochromatic radiances of the tungsten ribbon corresponding at $\lambda_{1}$ and $\lambda_{2}$ can be then computed with tungsten emissivity [18]. With the grey scale levels $N g_{\lambda_{1}}$ and $N g_{\lambda_{2}}$ obtained with the cameras, it is then possible to determinate the associated value of the sensitivity coefficient $K_{\lambda_{1}, \lambda_{2}}$.

This calibration procedure is performed for the different possible settings of the two-colour pyrometer, including:

- Three wavelength couples

- Three apertures

- Two lenses

- Exposure time varying from $25 \mu$ s to $1000 \mu \mathrm{s}$

For each configuration, the power supply is monitored in order to cover the whole possible temperature range. All the data associated to a same couple of wavelengths are plotted on a same graph. Fig. 3 shows such a graph for the couple $850 \mathrm{~nm} / 1000 \mathrm{~nm}$. In order to be suitable for any exposure time, these data are fitted with a power function:

$K_{\lambda_{1}, \lambda_{2}}=a t^{-b}+c$

The interpolated parameters obtained for all the wavelengths couple $\left(\lambda_{1}, \lambda_{2}\right)$ are summarized in Table 1 . For each couple, the maximum relative error between experimental data and corresponding interpolated value is also presented. The maximum one (3.02\%) is obtained for an exposure time of $250 \mu$ s and the $850 / 950 \mathrm{~nm}$ couple. The lower values of relative errors are obtained for $850 / 1000 \mathrm{~nm}$ and $850 / 905 \mathrm{~nm}$ couples. The function used to fit calibration results tends to an asymptotic behaviour.

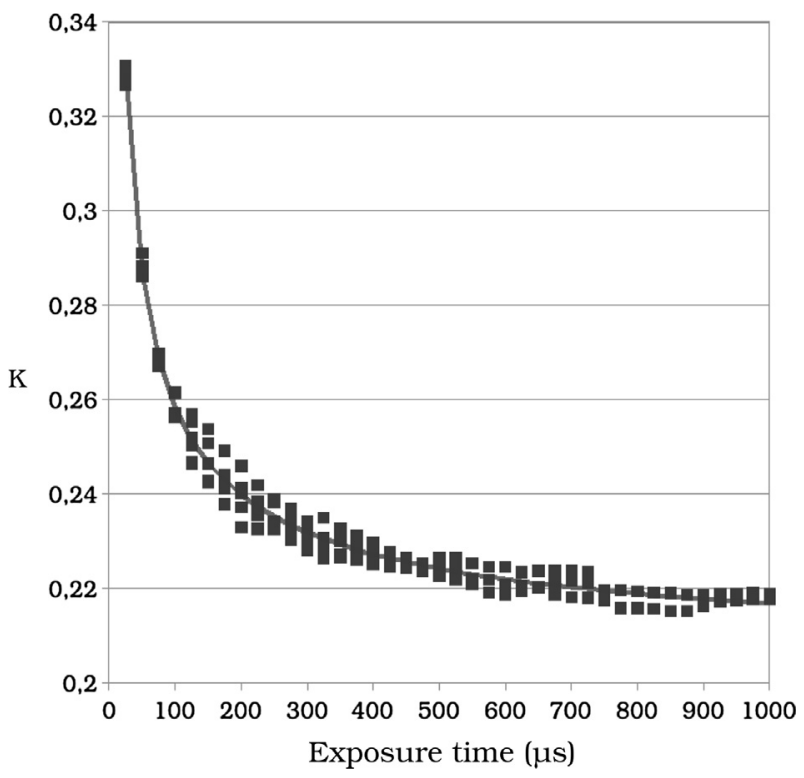

Fig. 3. Calibration procedure applied to sensitive coefficient $K_{850 / 1000}$. All the experimental data are synthesized in a same graph ( $\boldsymbol{\square})$, and the fitting relation (14) is plotted in solid line. 
Table 1

Calibration interpolation results obtained for the three wavelengths couples. Coefficients $a, b$ and $c$ are coefficients of Eq. (14) and $\frac{\Delta K}{K}$ is the maximum relative error between experimental data and the corresponding interpolated value.

\begin{tabular}{llll}
\hline$\lambda_{1} / \lambda_{2}$ & $850 / 1000$ & $850 / 950$ & $850 / 905$ \\
\hline $\mathrm{a}$ & 0.851 & 1.632 & 0.700 \\
$\mathrm{~b}$ & 0.615 & 0.858 & 0.520 \\
$\mathrm{c}$ & 0.202 & 0.332 & 0.621 \\
$\frac{\Delta K}{K}(\%)$ & 1.91 & 3.02 & 1.75 \\
\hline
\end{tabular}

According to the resulting curves such as the one presented in Fig. 3, it seems reasonable not to use low exposure time. Indeed, for exposure time below $200 \mu$ s, a very small variation requires an important variation of sensitivity coefficient $K$. Whereas, over $500 \mu$ s the sensitivity correction coefficient tends to stabilize. So, calibration over this exposure time is more accurate and so has less influence on measurement uncertainty.

\section{Uncertainty analysis}

The main sources of uncertainty lie in the model assumptions (grey body hypothesis), in the two-colour pyrometer device (interference filters) and in the conversion procedure from spectral radiance to grey scale levels. Each of these sources of uncertainty can be taken into account separately and discussed in regards with the wavelengths choice $\left(\lambda_{1}, \lambda_{2}\right)$. A logarithmic differentiation method has been used to evaluate temperature uncertainty, on the base of Eq. (5), in which the radiance ratio $\frac{L_{\lambda_{1}}}{L_{\lambda_{2}}}$ is replaced by $K_{\lambda_{1}, \lambda_{2}} N g_{\lambda_{1}, \lambda_{2}}$ where $N g_{\lambda_{1}, \lambda_{2}}=\frac{N g_{\lambda_{1}}}{N g_{\lambda_{2}}}$ is the grey scale ratio as presented in Section 3.2. After differentiating, some uncertainty parameters are inversely proportional to a coefficient, noted $F$, which can be written:

$\left.F=\ln K_{\lambda_{1}, \lambda_{2}}+\ln N g_{\lambda_{1}, \lambda_{2}}+5 \ln \lambda_{1}-5 \ln \lambda_{2}=\ln \quad K_{\lambda_{1}, \lambda_{2}} N g_{\lambda_{1}, \lambda_{2}} \frac{\lambda_{1}^{5}}{\lambda_{2}^{5}}\right)$

Combining Eqs. (5), (11) and (15), it appears that these uncertainty parameters are directly proportional to $\left|\frac{T \lambda_{1} \lambda_{2}}{C\left(\lambda_{2}-\lambda_{1}\right)}\right|$ with $C=\frac{h c}{k_{B}}$. The uncertainties are then directly proportional to the temperature of the actual object.

In order to evaluate the influence of wavelengths choice in the two-colours pyrometry method, reference configurations are fixed to calculate uncertainties.

The temperature is chosen at $3000 \mathrm{~K}$, which corresponds to the highest range of liquid metals temperatures, and grey scale levels are adapted to this value. Sensitivity correction coefficient $K_{\lambda_{1}, \lambda_{2}}$ is computed with parameters defined in Table 1 and with an exposure time of $500 \mu \mathrm{s}$, which is a frequently used setting in our experiments. The constant parameters used for uncertainties calculations are summarized in Table 2.

\subsection{Influence of grey body assumption}

The grey body assumption postulates that the ratio of the values of the emissivity at wavelengths $\lambda_{1}$ and $\lambda_{2}$ is equal to one.

Table 2

Constant parameters values used for uncertainties computations.

\begin{tabular}{llll}
\hline$\lambda_{1} / \lambda_{2}$ & $850 / 1000$ & $850 / 950$ & $850 / 905$ \\
\hline$K_{\lambda_{1}, \lambda_{2}}$ & 0.202 & 0.332 & 0.621 \\
$N g_{\lambda_{1}, \lambda_{2}}$ & 4.78 & 2.90 & 1.56 \\
\hline
\end{tabular}

$\left.\frac{\Delta T}{T}\right|_{\lambda, K_{\lambda_{1}, \lambda_{2}}}=\Delta \varepsilon_{R}\left|\frac{1}{F \varepsilon_{R}}\right|$

The other parameters $K_{\lambda_{1}, \lambda_{2}}$ and $\lambda=\left(\lambda_{1}, \lambda_{2}\right)$ are obtained from Table 2 . The uncertainties are then computed in function of different emissivity ratio $\varepsilon_{R}$ and an uncertainty of $\Delta \varepsilon_{R}=1 \%$ on emissivity ratio is chosen.

The results are presented in Table 3.

These results clearly show the influence of the couple of wavelengths, and specially that the values $850 / 1000 \mathrm{~nm}$ lead to the lower relative variation on the temperature measurement.

\subsection{Influence of monochromatic assumption}

The interference filters used in the two-colour pyrometer are narrowband filters with a bandwidth of $10 \mathrm{~nm}$. The uncertainty associated to each wavelength is $\Delta \lambda_{1}=\Delta \lambda_{2}=5 \mathrm{~nm}$. Considering the other parameters as constant, the relative variation of temperature due to the monochromatic assumption can be computed with:

$\left.\frac{\Delta T}{T}\right|_{\varepsilon_{R}, K_{\lambda_{1}, \lambda_{2}}}=\Delta \lambda_{1}\left|-\frac{5}{F \lambda_{1}}+\frac{1}{\lambda_{1}-\lambda_{2}}-\frac{1}{\lambda_{1}}\right|+\Delta \lambda_{2}\left|\frac{5}{F \lambda_{2}}-\frac{1}{\lambda_{1}-\lambda_{2}}+\frac{1}{\lambda_{2}}\right|$

The obtained values are presented in Table 4.

We can note that the better precision is obtained for spread wavelengths, but whatever the chosen couple, the effect on the measured temperature is not so important.

\subsection{Influence of conversion from radiance to grey scale level}

The conversion procedure integrates the complete process to convert spectral radiation to grey scale levels. It takes into account the whole optical path, the camera sensors and the associated uncertainties. The relative variation of measured temperature due to the variation of sensitive coefficient $K_{\lambda_{1}, \lambda_{2}}$ is:

$\left.\frac{\Delta T}{T}\right|_{\varepsilon_{R}, \lambda}=\Delta K_{\lambda_{1}, \lambda_{2}}\left|\frac{1}{F K_{\lambda_{1}, \lambda_{2}}}\right|$

The values of uncertainty $\Delta K_{\lambda_{1}, \lambda_{2}}$ are considered as the largest values determined in Table 1 for each couple of wavelengths. The effect on temperature variation is presented in Table 5. It appears that the wavelength choice directly influence the efficiency of the conversion procedure.

\section{Pure materials temperature measurements}

To assess the validity of the new device based on radiation theory, some melting tests were conducted on pure materials. The objective is to measure the temperature field at the surface of a liquid metal with different wavelength couples. By comparing the measured temperature with the melting temperature of pure material, the influence of wavelength can be determined. It is often argue that the two-colour pyrometer with the grey body assumption get rid of the knowledge of the emissivity. In order to investigate this advantage in large temperature range corresponding to temperature encountered in steel fusion process, four different

Table 3

Two colour pyrometer uncertainties on temperature measurement due to emissivity ratio (in \%).

\begin{tabular}{llll}
\hline$\lambda_{1} / \lambda_{2}$ & $850 / 1000$ & $850 / 950$ & $850 / 905$ \\
\hline$\left.\frac{\Delta T}{T}\right|_{\lambda, K_{\lambda_{1}, \lambda_{2}}}$ & 1.18 & 1.68 & 2.90 \\
\hline
\end{tabular}


Table 4

Two colour pyrometer uncertainties due to optical filter bandwidth (in \%).

\begin{tabular}{llll}
\hline$\lambda_{1} / \lambda_{2}$ & $850 / 1000$ & $850 / 950$ & $850 / 905$ \\
\hline$\left.\frac{\Delta T}{T}\right|_{\varepsilon_{R}, K_{\lambda_{1}, \lambda_{2}}}$ & 1.34 & 1.73 & 2.80 \\
\hline
\end{tabular}

Table 5

Two colour pyrometer uncertainties due to spectral calibration uncertainties (in \%).

\begin{tabular}{llll}
\hline$\lambda_{1} / \lambda_{2}$ & $850 / 1000$ & $850 / 950$ & $850 / 905$ \\
\hline$\Delta K$ & 0.00385 & 0.01 & 0.0109 \\
$\left.\frac{\Delta T}{T}\right|_{\varepsilon_{R}, \lambda}$ & 2.25 & 5.07 & 5.08 \\
\hline
\end{tabular}

materials (iron Fe, vanadium $\mathrm{V}$, niobium $\mathrm{Ni}$ and tantalum $\mathrm{Ta}$ ) were chosen to cover this temperature range.

\subsection{Experimental set-up}

The experimental set-up is shown in Fig. 4. The metal coupons were cylindrical rods of $2 \mathrm{~mm}$ of diameter and their length could reach $75 \mathrm{~mm}$. Coupons were fixed in a small chuck set into a glass tube which is transparent to radiation $500-1100 \mathrm{~nm}$. Argon gas flowed through the glass tube $(40 \mathrm{l} / \mathrm{min})$ and insured the shielding of the liquid metal.

The metal rod was heated by a disk laser Trumpf operating at $1030 \mathrm{~nm}$. The laser provided an uniform intensity distribution on the top flat surface of the rod. The energy led to the fusion of the material. Depending on the material, different heating time and power were set in order to have a large volume of liquid metal to be able to observe the solidification. Due to thermal boundary conditions with the clamping device and with the thermal exchange with the active flow of argon, it is difficult to set the process parameters and they were experimentally determined. The heat input is controlled by the power of the laser and the impulsion time. The process parameters for each pure material are given in Table 6.

The two-colour pyrometer was tilted at 45 degrees relative to the rod axis and a framerate of $100 \mathrm{fps}$ was chosen to record the solidification. The melting temperature and some physical properties are given in Table 7.

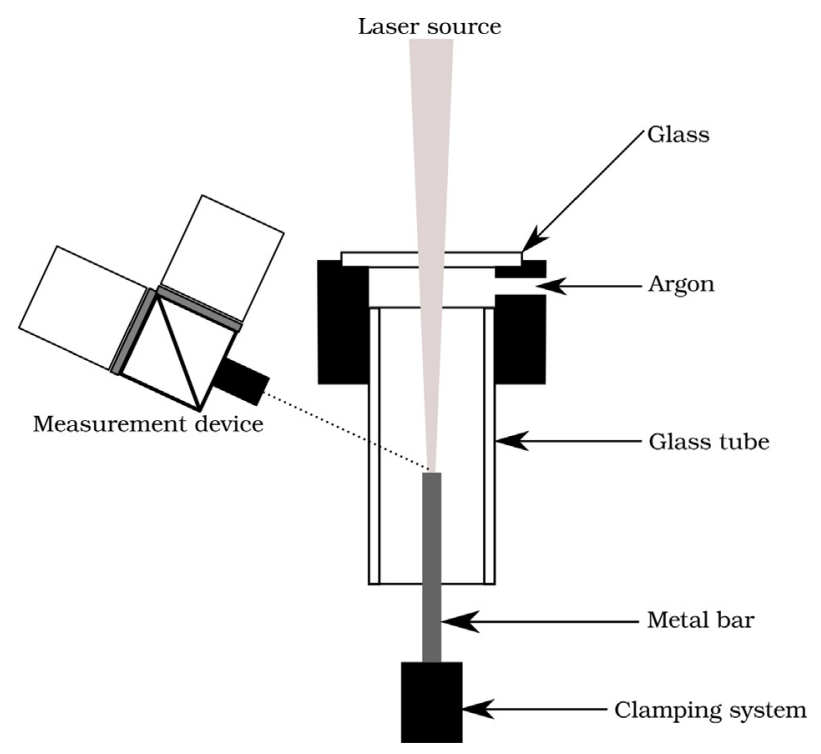

Fig. 4. Schematic illustration of pure material melting tests.
The Wien's assumption is adequate for the four materials. Around the melting temperature, the emissivity is in general dependent on the wavelength. Previous works [21-23,2] studied the emissivity and approached it with a linear function $\varepsilon=a \lambda+b$ around the melting temperature. Values for coefficients $a$ and $b$ are given in Table 7 . The different coefficients demonstrate that the emissivity can not be considered as constant. This will be investigated by comparing temperature results coming from grey body assumption and those integrating the ratio of emissivity at the two wavelengths of the pyrometer.

The tests were repeated on the four materials with three filter couples:

- $850 \mathrm{~nm}$ and $1000 \mathrm{~nm}$

- $850 \mathrm{~nm}$ and $950 \mathrm{~nm}$

- $850 \mathrm{~nm}$ and $905 \mathrm{~nm}$

This represents a total of twelve different temperature measurement configurations which allow the evaluation of the overall system efficiency.

\subsection{Raw images during solidification}

Fig. 5 shows the raw pictures of an iron rod during the cooling process just after the stop of the heating due to the laser beam. For this material and for process parameters, the iron liquid phase on the top of the rod has spherical cap shape. Three different steps occur during the solidification in this figure. In the first step, the spherical cap is completely liquid and wets on the rod. This step lasts around $30 \mathrm{~ms}$. During this phase, an impurity (white phase) is visible on the surface. This impurity has a motion indicating a fluid flow inside the spherical cap. Impurity velocity is estimated around $0.15 \mathrm{~m} / \mathrm{s}$. This fluid motion can lead to a blurring effect. A second step consists in the stop of the liquid flow and the start of the solidification. In the iron case, the solidification begins from the rod and continues to the top of the spherical cap. The solidification front is observed with a slight discontinuity in grey levels of the raw images. When the front is advancing, it can also be observed, on the side of the spherical cap, a change in the geometry due to the difference of density between liquid and solid. At the end, the top cap is still liquid and it is the last part to solidify. The scenario is almost the same for vanadium. It is more complicated to see the advancing front for niobium and even impossible to see it for tantalum rod. This fact will be discussed after temperature surface will be measured.

Only for iron (not other pure materials), some foreign particles appear when the solidification front reaches the middle of the spherical cap.

For iron and vanadium, a high wetting angle is visible. This implies a large volume of melted material (measured around $9 \mathrm{~mm}^{3}$ ). For niobium and tantalum, the wetting angle is smaller in relation with a low volume of melted material (estimated around $2.5 \mathrm{~mm}^{3}$ ). For these four materials, the thermal diffusivity and the Stephan numbers have the same order of magnitude. It means that more the volume of melted material is important more the solidification time is higher. With higher solidification time, it is easier to detect the solidification front. Depending on the material, the solidification is visible between 10 and 2 images depending on the solidification velocity. It is important to detect the solidification front because when solidification occurs there is a release of heat and despite the high conduction in the rod and the radiation to the ambient, the temperature stays constant and is around the melting temperature. This melting temperature can be used to assess the designed temperature sensor. If the front is 
Table 6

Laser melting parameters.

\begin{tabular}{lllll}
\hline Material & $\mathrm{Fe}$ & $\mathrm{V}$ & $\mathrm{Nb}$ & $\mathrm{Ta}$ \\
\hline Impulsion time (ms) & 2000 & 2000 & 2400 & 3000 \\
Power (W) & 320 & 320 & 320 & 380 \\
\hline
\end{tabular}

Table 7

Material properties. The values are given by Good fellow. The emissivity function of wavelength are extracted from the literature [21-23,2].

\begin{tabular}{|c|c|c|c|c|}
\hline Thermal properties & $\mathrm{Fe}$ & $\mathrm{V}$ & $\mathrm{Nb}$ & $\mathrm{Ta}$ \\
\hline Temperature (K) & 1810 & 2183 & 2742 & 3269 \\
\hline Latent heat $\left(\mathrm{kJ} \mathrm{kg}^{-1}\right)$ & 272 & 345 & 290 & 174 \\
\hline Capacity $\left(\mathrm{J} \mathrm{K}^{-1} \mathrm{~kg}^{-1}\right)$ & 444 & 486 & 268 & 140 \\
\hline Conductivity ( $\mathrm{W} \mathrm{K}^{-1} \mathrm{~m}^{-1}$ ) & 80.4 & 30.7 & 53.7 & 57.5 \\
\hline Density $\left(\mathrm{kg} \mathrm{m}^{-3}\right)$ & 7870 & 6100 & 8570 & 16,600 \\
\hline \multicolumn{5}{|l|}{ Emissivity } \\
\hline $\mathrm{a}\left(10^{-4} \mathrm{~nm}^{-1}\right)$ & -2.602 & -0.681 & -1.76 & -1.72 \\
\hline $\mathrm{b}$ & 0.602 & 0.456 & 0.492 & 0.532 \\
\hline
\end{tabular}

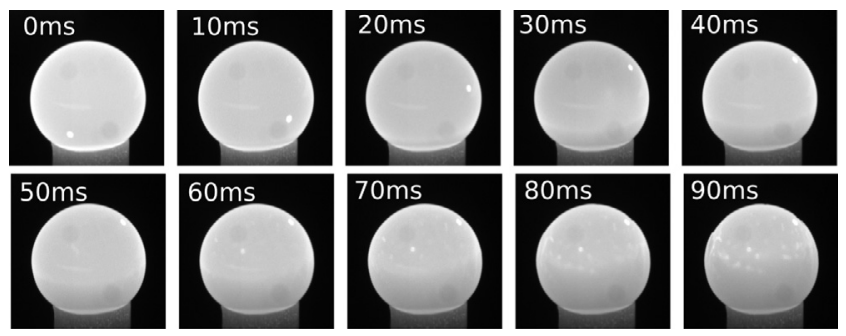

Fig. 5. Images obtained during solidification of pure iron for the camera with a $850 \mathrm{~nm}$ narrow band filter.

advancing too quickly, the constant temperature cannot be measured.

\subsection{Global uncertainty for pure material}

Before presenting temperature results, the global uncertainty of the two-colour pyrometry method taking into account emissivity, temperature level and conversion of radiance to grey level are computed for the different pure materials. Table 8 presents the results in function of wavelength couples and material.

Table 8 demonstrates that the wavelength couple $850 / 1000 \mathrm{~nm}$ seems to be the most efficient in term of uncertainties. It is interesting to notice that the uncertainties are combined. The highest relative error is not for the material with the highest melting temperature.

\subsection{Surface temperature results}

The set-up is used to estimate surface temperature. The results for a melting experiment on vanadium is presented in order to explain the methodology to assess the measurement. The raw images for two narrow band filters are correlated in order to minimize the difference in grey level with Eq. (9). The two images are

Table 8

Two colour pyrometer global uncertainties $\Delta T / T$ (in \%).

\begin{tabular}{llll}
\hline$\lambda_{1} / \lambda_{2}$ & $850 / 1000$ & $850 / 950$ & $850 / 905$ \\
\hline $\mathrm{Fe}$ & 6.21 & 9.64 & 15.05 \\
$\mathrm{~V}$ & 8.06 & 12.11 & 19.69 \\
$\mathrm{Ni}$ & 7.24 & 11.01 & 17.64 \\
$\mathrm{Ta}$ & 6.90 & 10.56 & 16.80 \\
\hline
\end{tabular}

used to compute the temperature based on Eq. (5) with grey emissivity assumption $\left(C_{f}=1\right)$ or with a compensation of emissivity $\left(C_{f} \neq 1\right)$. These equations are then applied at each pixel and then the result is filtered within a temperature range. A result is shown on Fig. 6 for a vanadium melting test.

In Fig. 6, the temperature is shown just after the stop of the laser heating. Despite the shielding, an impurity modifies the measurement near the top of the cap, nevertheless, the computed temperature field is continuous.

The overall evolution of the solidification is similar to the iron. The solidification starts from the rod and goes to the top of the spherical cap. The higher temperature remains located at the top of the spherical cap. The lower parts have cooled down. The difference is clearly visible but the solid liquid interface can not clearly be distinguished. This result is representative of surface temperature measurement during the solidification of the rod. To investigate the temperature more deeply and analyse the solidification behaviour, two kinds of extraction are then performed.

The first one corresponds to three probes located respectively near the rod, in the middle of the spherical cap and at the top. The temperature evolution is plotted with respect to time for the three probes. For results in Fig. 6, the evolution of the probes are depicted in Fig. 7.

All the probes start almost from the same temperature. The probe near the rod cools down directly. The two other probes stay almost at a constant temperature and then cool down. The probe at the middle starts first and soon after the top probe cools down also. It is due to the rapid advance of the solidification front due to the decrease of volume of the liquid phase. A slight heating (increase of 10 degrees) is measured for the top probe but it is difficult to argue if it is a phenomena due to the latent heat release during solidification or due to a perturbation in the experimental method or due to the fluid motion. For these two probes, the constant temper-
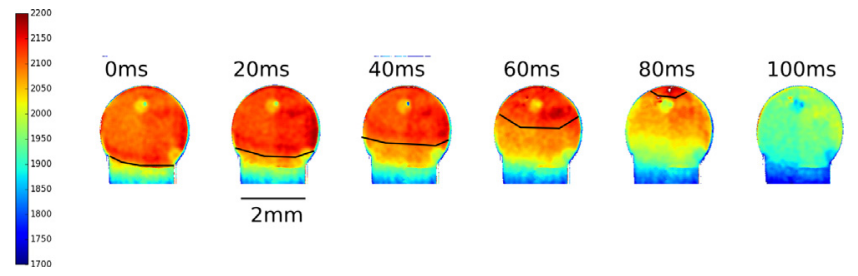

Fig. 6. Temperature measurement with $850-1000 \mathrm{~nm}$ narrow band filter couple for vanadium melting test and grey emissivity assumption. 


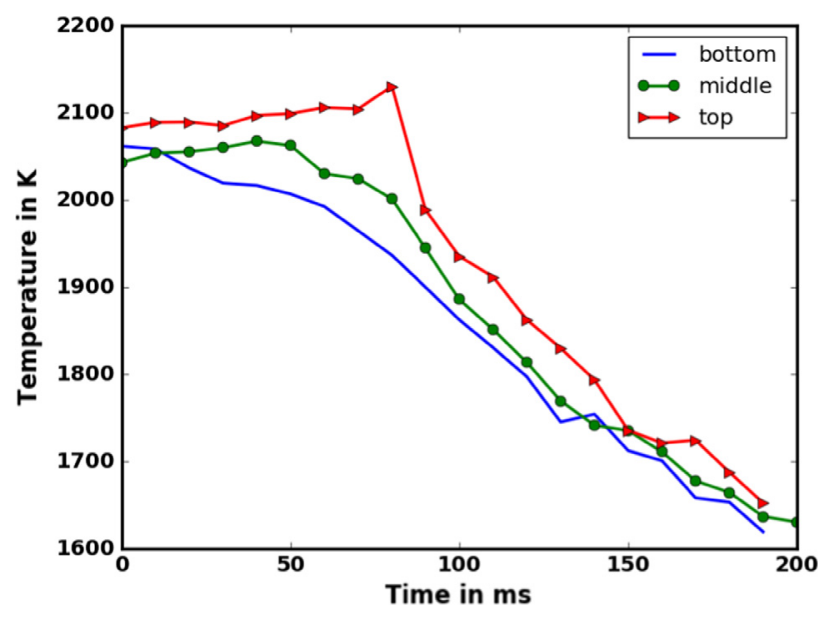

Fig. 7. Temperature evolution at three locations (near the rod, the middle and the top) for surface temperature field of Fig. 6 .

ature can be clearly estimated. Results in Fig. 7 give a melting temperature of the vanadium around $2069 \mathrm{~K}$. It is interesting to remark that when the whole spherical cap is solidified the temperature becomes homogeneous and all the probes give almost the same temperature. This result is consistent with the solidification mechanism.

For pure vanadium, the emissivity depends on the wavelength and a compensation factor can be taken into account to release the grey emissivity assumption. For the couple wavelength 850 $1000 \mathrm{~nm}$, the correction factor is around 1.032 . For the configuration presented in Fig. 6, this tends to modify the temperature field but the trends are identical. From the temperature field and the probes, the constant melting temperature is measured around $2124 \mathrm{~K}$. In the literature, the vanadium melting temperature is given at $2183 \mathrm{~K}$. This leads for the grey emissivity assumption to a relative error of $5 \%$ and $2.5 \%$ for the compensation of emissivity. This technique is repeated for the four pure materials to measure melting temperature. The procedure gives results for iron, vanadium and niobium but fails for tantalum. For tantalum, the liquid volume is too small and probably the too high temperature leads to high radiation loss, the solidification front is not clearly visible. Table 9 presents the obtained data.

The second extraction is realized in order to see the evolution of the temperature field along a line on the surface. A segment in the liquid phase is divided with 20 points. The segment starts from the top of the spherical cap and stops inside the rod. The profile of temperature along this segment is plotted versus time. Fig. 8 presents the results for tantalum.

The temperature profiles in Fig. 8 demonstrate the fast solidification of the tantalum specimen. Between the first image just after the stop of the heating and the second one, the temperature drops under the melting temperature. It is then difficult to see the liquid/solid interface and the constant melting temperature. This explains the fact that it is difficult to use the tantalum melting experiment in the assessment of the set-up. The profiles reveal also a change in solidification mode for tantalum. For iron, vanadium and niobium, the solidification starts from the rod and a front advances until the top of the spherical cap. For tantalum, surface radiation is more important due to the higher temperature and this seems to lead to a solidification from the external skin to the core of the liquid phase.

\subsection{Analysis and comparison of results}

These experiments were designed to test the experimental setup. Results were obtained for iron, vanadium and niobium. Two kind of results were obtained: with constant emissivity on one hand and with a correction factor taking into account the variation of emissivity with respect to wavelength couple on the other hand. For the whole measurements, the computations of the melting temperature are good. The computed temperature with grey emissivity assumptions are within a maximum of ten percent relative error and are often around 5\%. The computed temperature with emissivity wavelength compensation based on literature are excellent. This means that the main deviation of the temperature with grey emissivity assumption comes from the hypothesis of a constant emissivity for the two wavelengths. These results assess of the procedures presented in the calibration of the proposed device. This trends is confirmed by the computed temperatures with GE assumption: the couple of wavelengths that gives the less error is the one with the closest wavelength (850-905 nm) and probably the less emissivity difference. If the emissivity function of wavelength is known, the compensation can be introduced. In this case, the couple $850-1000 \mathrm{~nm}$ gives the best result. This comes probably from the best sensitivity difference between the two sensors at these wavelengths.

It is interesting to see that for the highest melting temperature, the error is higher when wavelength difference is large for grey emissivity assumption. The integration of emissivity compensation modified the temperature level. The compensation is integrated in the log term of Eq. (5) and it is not a proportional correction. With this in mind and with the difference of results, it means that the trends can be given by the grey assumption context.

For the different results, the relative error determined with the GE assumption are within the global uncertainties determined. The measured melting temperature are most of the time below the melting temperature given by the literature.

One important point for the design set-up is that the technique does not need any calibration for the different melting pure material tests even if the surface is a spherical cap and not flat. This means that the device can be used on complex liquid free surface

Table 9

Melting temperatures for pure materials and three different wavelength couples. GE stands for grey emissivity assumption and EC for emissivity compensation.

\begin{tabular}{|c|c|c|c|c|}
\hline Temperature & $\mathrm{Fe}$ & V & $\mathrm{Nb}$ & $\mathrm{Ta}$ \\
\hline GE $850-1000 \mathrm{~nm}$ couple $(K)$ & 1695 & 2069 & 2464 & - \\
\hline Relative error (\%) & 6.3 & 5 & 10 & - \\
\hline GE $850-950 \mathrm{~nm}$ couple $(K)$ & 1685 & 2045 & 2408 & - \\
\hline Relative error (\%) & 7 & 6 & 12 & - \\
\hline GE 850-905 nm couple $(K)$ & 1635 & 2040 & 2603 & - \\
\hline Relative error (\%) & 9 & 6.5 & 5 & - \\
\hline EC $850-1000 \mathrm{~nm}$ couple $(K)$ & 1821 & 2124 & 2694 & - \\
\hline Relative error (\%) & 0.6 & 2.7 & 1.7 & - \\
\hline EC $850-950 \mathrm{~nm}$ couple $(K)$ & 1804 & 2095 & 2583 & - \\
\hline Relative error (\%) & 0.3 & 4 & 5.7 & - \\
\hline EC $850-905 \mathrm{~nm}$ couple $(K)$ & 1745 & 2088 & 2800 & - \\
\hline Relative error (\%) & 3.6 & 4.3 & 2. & - \\
\hline
\end{tabular}




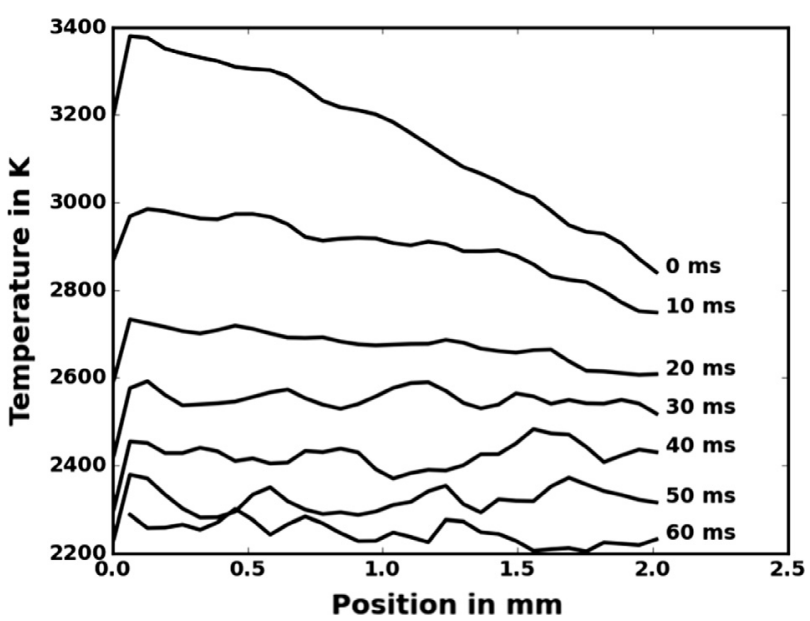

Fig. 8. Temperatures along a line going from the top to the bottom of the tantalum rod. Each line is the temperature profile at a specific time. Time $0 \mathrm{~ms}$ correspond to laser heating stop.

as it can be encountered during joining process, even with unknown emissivity behaviour.

\section{Conclusion}

The method proposed here to measure liquid metal temperatures shows a good accuracy. Wavelength choice is the key parameter to considerer to set up the temperature range. Pure material melting tests showed the efficiency of the measurement. The grey body hypothesis applied here seems valid for these wavelengths couples. In fact, the absolute error does not significantly change even if the chemical composition of the material changes. The sensitivity of the method regarding to the calibration process seems also not to be affected. A complete geometrical calibration of the device is necessary to ensure no defects on final temperature fields. Furthermore, the spectral calibration determines the correction coefficient which directly influences temperature estimation. This coefficient has to be determined precisely in function of all the optical set up. We also observed a high sensitivity of the device to optical cleanliness. A tiny difference between the two optical way can cause a huge lack of information.

Measurement of temperature field of liquid metals could be used in all industrial processes which involve high heat transfers. Fields such as welding or smelting works need to control heat transfer. Temperature of liquid material could be used as an interesting indicator of the process quality.The tests presented here are done in a laboratory configuration. In these conditions this method gave a precise accuracy. But, the atmosphere is more disturbed around industrial manufacturing processes. So, the influence of external perturbations have to studied. These external perturbations are mainly composed of fumes and reflections from the environment.

\section{Acknowledgement}

The authors are grateful for the support of the laboratory "Procédés et Ingnierie en Mécanique et Matériaux". The authors would like to specially thank Morgan Dal and Frédéric Coste for their help.

\section{References}

[1] C. Meola, G.M. Carlomagno, Recent advances in the use of infrared thermography, Meas. Sci. Technol. 15 (9) (2004) R27.

[2] H. Watanabe, M. Susa, H. Fukuyama, K. Nagata, Phase (liquid/solid) dependence of the normal spectral emissivity for iron, cobalt, and nickel at melting points, Int. J. Thermophys. 24 (2) (2003) 473-488.

[3] B. Wilthan, C. Cagran, G. Pottlacher, E. Kaschnitz, Normal spectral emissivity at $684.5 \mathrm{~nm}$ of the liquid binary system Fe-Ni, Monat. Chem./Chem. Mon. 136 (11) (2005) 1971-1976.

[4] H. Schöpp, A. Sperl, R. Kozakov, G. Gött, D. Uhrlandt, G. Wilhelm, Temperature and emissivity determination of liquid steel S235, J. Phys. D: Appl. Phys. 45 (23) (2012) 235203

[5] M. Muller, R. Fabbro, H. El-Rabii, K. Hirano, Temperature measurement of laser heated metals in highly oxidizing environment using 2D single-band and spectral pyrometry, J. Laser Appl. 24 (2) (2012).

[6] J. Thevenet, M. Siroux, B. Desmet, Measurements of brake disc surface temperature and emissivity by two-color pyrometry, Appl. Therm. Eng. 30 (6) (2010) 753-759.

[7] K. Yamazaki, E. Yamamoto, K. Suzuki, F. Koshiishi, K. Waki, S. Tashiro, M. Tanaka, K. Nakata, The measurement of metal droplet temperature in GMA welding by infrared two-colour pyrometry, Weld. Int. 24 (2) (2010) 81-87.

[8] M. Tanaka, K. Waki, S. Tashiro, K. Nakata, E. Yamamoto, K. Yamazai, K. Suzuki, Visualization of 2D temperature distribution of molten metal in arc welding process, Trans. JWRI 38 (2009) 1-4.

[9] A. Hijazi, S. Sachidanandan, R. Singh, V. Madhavan, A calibrated dualwavelength infrared thermometry approach with non-greybody compensation for machining temperature measurements, Meas. Sci. Technol. 22 (2) (2011) 025106

[10] V. Tank, H. Dietl, Multispectral infrared pyrometer for temperature measurement with automatic correction of the influence of emissivity, Infrared Phys. 30 (4) (1990) 331-342.

[11] C.-D. Wen, Investigation of steel emissivity behaviors: examination of multispectral radiation thermometry (MRT) emissivity models, Int. J. Heat Mass Transf. 53 (9) (2010) 2035-2043.

[12] B. Tsai, R. Shoemaker, D. DeWitt, B. Cowans, Z. Dardas, W. Delgass, G. Dail Dual-wavelength radiation thermometry: emissivity compensation algorithms, Int. J. Thermophys. 11 (1) (1990) 269-281.

[13] C.-D. Wen, I. Mudawar, Emissivity characteristics of roughened aluminum alloy surfaces and assessment of multispectral radiation thermometry (MRT) emissivity models, Int. J. Heat Mass Transf. 47 (17) (2004) 3591-3605.

[14] L. Del Campo, R.B. Pérez-Saez, M.J. Tello, Iron oxidation kinetics study by using infrared spectral emissivity measurements below $570{ }^{\circ} \mathrm{C}$, Corros. Sci. 50 (1) (2008) 194-199.

[15] S. MATTEÏ, Rayonnement thermique des matériaux opaques, Techniques de l'ingénieur Transferts thermiques (ref. article: be8210). Avaliable from: $<$ arXiv:base documentaire: TIB214DUO>.

[16] K. Yamazaki, E. Yamamoto, K. Suzuki, F. Koshiishi, S. Tashiro, M. Tanaka, K. Nakata, Measurement of surface temperature of weld pools by infrared two colour pyrometry, Sci. Technol. Weld. Joi. 15 (2009) 40-47.

[17] Gary Bradski, A. Kaehler, Computer Vision with the OpenCV Library, O-Reilly, 2008.

[18] R.D. Larrabee, The Spectral Emissivity and Optical Properties of Tungsten, Optical Society of America, 1959.

[19] D.R. Lide, CRC Handbook of Chemistry and Physics, CRC Press, 2004.

[20] C. De Izarra, J.-M. Gitton, Calibration and temperature profile of a tungsten filament lamp, Eur. J. Phys. 31 (4) (2010) 933.

[21] A. Cezairliyan, J.L. McClure, A.P. Miiller, Radiance temperatures of tantalum at its melting point by a pulse-heating technique, High Temp. High Press. 25 (6) (1993) 649-656.

[22] A. Cezairliyan, A. Miiller, Radiance temperatures (in the wavelength range $522-906 \mathrm{~nm}$ ) of niobium at its melting point by a pulse-heating technique, Int. J. Thermophys. 13 (1) (1992) 39-55.

[23] J. McClure, A. Cezairliyan, Radiance temperatures (in the wavelength range 525 to $906 \mathrm{~nm}$ ) of vanadium at its melting point by a pulse-heating technique, Int. J. Thermophys. 18 (1) (1997) 291-302. 